\title{
Determinação Eletroanalítica de Íons Sódio com Eletrodo de Pasta de Carbono Modificado com Óxido de Níquel
}

\author{
Mônica Libânia Mendonça Firmino* (Mestranda do Programa de Pós-Graduação em Ciências Naturais \\ na Universidade do Estado do Rio Grande do Norte - UERN); \\ Thiago Mielli B. F. Oliveira (Professor do Programa de Pós-Graduação em Ciências Naturais na \\ Universidade do Estado do Rio Grande do Norte - UERN) \\ *E-mail: monica_nina86@hotmail.com
}

\begin{abstract}
Resumo: Uma dieta rica em íons $\mathrm{Na}^{+}$tem sido associada a problemas cardíacos, disfunções hormonais, alergias, entre outras patologias. Convencionalmente, o monitoramento deste cátion em diferentes matrizes vem sendo feito por métodos cromatográficos e espectroscópicos, embora apresentem limitações quanto ao custo, interferência e tempo de análise. Este trabalho tem por objetivo desenvolver um método eletroanalítico alternativo, utilizando um eletrodo de pasta de carbono quimicamente modificado com um filme de óxido de níquel (NiOx/EPC), que permita a detecção deste cátion em diferentes matrizes e apresente um melhor custo-benefício em comparação ao métodos convencionais. A voltametria de onda quadrada (VOQ) foi utilizada na etapa de quantificação, após a otimização dos parâmetros que controlam a resposta eletroquímica nesta técnica (frequência de $100 \mathrm{~Hz}$, amplitude de $30 \mathrm{mV}$ e incremento de varredura de $3 \mathrm{mV}$ ). Considerando o limite de deteç̧ão de $9,50 \times 10^{-7} \mathrm{~mol} \mathrm{~L}^{-1}$ e o baixo desvio padrão relativo obtido para os testes de precisão das medidas $(<7,0 \%)$, a associação entre NiOx/EPC e VOQ, pode apresentar resultados promissores na eletroanálise e controle de qualidade de amostras supostamente contaminadas por este cátion.
\end{abstract}

Palavras-chave: Íons sódio; Eletrodos quimicamente modificados; Óxido de níquel; Determinação eletroanalítica.

\section{Introdução}

O sódio é um elemento químico de fundamental importância para o organismo, pois este auxilia na regulação osmótica dos fluidos corporais, atua na condução de impulsos nervosos e na contração muscular. A forma ionizada deste elemento pode ser encontrado nos alimentos sob diferentes associações, embora sua ampla aplicação como condimento na culinária, na forma de cloreto de sódio ou "sal de cozinha" - $\mathrm{NaCl}_{(\mathrm{s})}$, tenha tornado esta a mais importante fonte de íons $\mathrm{Na}^{+}$para a dieta diária. Os cuidados com a ingestão excessiva deste micronutriente se devem às inúmeras patologias e efeitos colaterais que podem ser desencadeados, como hipertensão, disfunções hormonais, aborto espontâneo, doenças de pele, infecção respiratória aguda, complicações renais, doenças diarreicas, além do risco de morte em muitos casos.

Outra importante fonte de íons $\mathrm{Na}^{+}$, apesar de pouco enfatizada, são as águas utilizadas para o consumo. O mecanismo de acesso deste cátion aos corpos hídricos se dá por diferentes processos naturais e antrópicos como, por exemplo, através do descarte de efluente domésticos e industriais sem tratamento, lixiviação de águas utilizadas para a irrigação (FIRMINO et al., 
2015), erosão e dissolução de formações rochosas em contato com o aquífero, infiltração de águas salinas oriundas de correntes marítimas (CHAUDHURI e ALE, 2014), percolação do solo (MACHIWAL e JHA, 2015; KELLY, PANNO e HACKLEY, 2012), entre outros. Deste modo, há um grande interesse no desenvolvimento de métodos para a detecção e quantificação deste cátion nessas matrizes, como forma de prevenir possíveis distúrbios na saúde dos seres vivos em decorrência da exposição crônica.

Entre os métodos padrão mais utilizados para a análise deste cátion, encontram-se a fotometria de chama, espectroscopia de absorção e emissão atômica e a cromatografia de íons (APHA, 2005). Entretanto, os métodos cromatográficos demandam uma dispendiosa infraestrutura, enquanto os métodos espectroscópicos apresentam maior susceptibilidade a interferências químicas e físicas e, normalmente, requerem etapas de pré-tratamento que aumentam o tempo de análise (QUINTINO, 2003; CARMO, SILVA, STRADIOTTO, 2004; MAFTALE, NYOKONG, 1996; PERREIRA, SANTOS, KUBOTA, 2002). Assim, muitos laboratórios têm implantado os métodos eletroanalíticos em suas rotinas, por serem mais práticos, permitirem análises mais rápidas e com custo acessível, além de possibilitarem a miniaturização e portabilidade dos equipamentos. Os métodos eletroanalíticos também são mais robustos e menos susceptíveis a interferentes naturais e antrópicos presentes nas amostras, possibilitando, em alguns casos, experimentos in situ, análises em amostras coloridas e/ou na presença de partículas suspensas (OLIVEIRA, 2013; CARMO, SILVA, STRADIOTTO, 2004).

Com o avanço nas pesquisas com materiais condutores, uma grande variedade de sensores vem sendo desenvolvidos com o intuído de aumentar a sensibilidade, estabilidade e seletividade na eletroanálise, como destaque para os eletrodos quimicamente modificados. $\mathrm{O}$ termo eletrodo quimicamente modificado (EQM) foi utilizado inicialmente na eletroquímica por Murray e colaboradores em 1975, para caracterizar eletrodos com espécies quimicamente ativas, imobilizadas em sua superfície, com objetivo de pré-estabelecer e controlar a natureza físicoquímica da interface eletrodo/solução (SOUZA, 1997). Os EQMs são simples, bastante versáteis e apresentam algumas vantagens sobre os eletrodos convencionais, como eletrocatálise, resistência a passivação, aumento da eletroatividade e melhoria da cinética de transferência eletrônica (QUINTINO, 2003; CARMO, SILVA, STRADIOTTO, 2004; MAFTALE, NYOKONG, 1996; PERREIRA, SANTOS, KUBOTA, 2002).

Dentre os diferentes agentes modificadores de superfícies eletródicas, encontram-se os hexacianoferratos, por possuírem a capacidade de formar complexos com uma grande variedade de metais de transição (ABBASPOUR, KHAJEHZADEH, GHAFFARINEJAD, 2009). As reações eletroquímicas são baseadas em duas características principais: a presença de pares redox ativos de íons metálicos e a presença de cavidades que possam alojar íons metálicos, como o $\mathrm{Na}^{+}, \mathrm{K}^{+}$e $\mathrm{Li}^{+}$(VINCETINI, 2009). Os complexos de hexacianoferrato de níquel (NiHCF), por exemplo, possuem uma estrutura cúbica, onde os átomos de Fe e Ni se encontram ligados aos grupos cianeto. O centro do cubo é ocupado por cátions de metais alcalinos para manter a neutralidade de cargas (SCHNEEMEYER, SPENGLER, MURPHY, 1985).

Em relação à determinação eletroanalítica de íons $\mathrm{Na}^{+}$, os ensaios são geralmente feitos por potenciometria, embora haja limitações para a aplicação direta devido às limitações do método com relação ao pH e força iônica do meio. Apesar de pouco explorados para este fim, Chen et al. (2009) mostraram a eficiência de EQMs, formados por um eletrodo de ouro quimicamente modificado com Ni-HCF, para a determinação de $\mathrm{Na}^{+}$em amostras de soro e plasma sanguíneo de seres humanos, sem nenhum tratamento prévio das amostras, para uma faixa de concentração de $1,0 \times 10^{-4}$ a $1,0 \mathrm{~mol} \mathrm{~L}^{-1}$. Em face do exposto e diante da escassez de trabalhos na literatura na área dos EQMs para íons $\mathrm{Na}^{+}$, o presente trabalho tem por objetivo desenvolver um método eletroanalítico alternativo para a detecção e quantificação deste cátion, utilizando um eletrodo de pasta de carbono modificado com óxido de níquel (NiOx/EPC) como sensor de trabalho, visando sua aplicação em amostras de interesse biológico e ambiental. 


\section{Metodologia}

\subsection{Reagentes e soluções}

Todos os reagentes utilizados neste estudo foram de pureza analítica. Uma solução $1,0 \times 10^{-3}$ mol $\mathrm{L}^{-1}$ de $\mathrm{Na}^{+}$foi preparada em meio aquoso e utilizada como solução estoque do analito. Para os ensaios eletroanalíticos, utilizou-se uma solução de hidróxido de sódio $0,1 \mathrm{~mol} \mathrm{~L}^{-1}$ como eletrólito de suporte. Todas as soluções foram preparadas a temperatura ambiente $\left(25^{\circ} \mathrm{C}\right)$ e utilizando água ultrapura, obtidas de um sistema Purelab Milli-Q (Analítica).

\subsection{Instrumentação}

Os experimentos eletroquímicos foram realizados em um Potenciostato $\mu$-Autolab, modelo Type III (Methrom) e controlado por um computador, usando o programa NOVA versão 1.10, acoplada a uma célula eletroquímica com capacidade para $10 \mathrm{~mL}$, composta por um sistema de três eletrodos: (1) o NiOx/EPC como eletrodo de trabalho, (2) um eletrodo de platina como eletrodo auxiliar e (3) um eletrodo de $\mathrm{Ag} / \mathrm{AgCl} / \mathrm{C}_{\text {saturado }}$ como eletrodo de referência.

\subsection{Construção do eletrodo modificado}

Para a construção do EQM, utilizou-se um eletrodo de pasta de carbono (pó de grafite ultrapuro e nujol, na proporção $75: 25 \mathrm{~m} / \mathrm{m}$ ) como suporte eletródico. O material foi embutido na cavidade de um tubo de polietileno, com 5,0 mm de diâmetro, sendo o contato elétrico estabelecido por um fio de aço-inox. Posteriormente, este dispositivo foi imerso em uma solução mista de $\mathrm{K}_{3} \mathrm{Fe}(\mathrm{CN})_{6}$ a $1,0 \times 10^{-3} \mathrm{~mol} \mathrm{~L}^{-1}, \mathrm{NiCl}_{2}$ a $1,0 \times 10^{-3} \mathrm{~mol} \mathrm{~L}^{-1}$ e $\mathrm{KCl}$ a $0,2 \mathrm{~mol} \mathrm{~L}^{-1}$, com o intuito de eletrodepositar hexacianoferrato de níquel sobre a superfície eletródica, através de varreduras consecutivas de potencial $(0,0-1,0 \mathrm{~V})$ a uma velocidade de $50 \mathrm{mV} \mathrm{s}^{-1}$. O óxido de níquel foi eletrossintetizado in situ, fazendo uma nova sequência de varreduras de potencial a $50 \mathrm{mV} \mathrm{s}^{-1}$, em meio de $\mathrm{NaOH} 0,1 \mathrm{~mol} \mathrm{~L}^{-1}$, produzindo o EQM de trabalho (NiOx/EPC).

\subsection{Determinação eletroanalítica}

A determinação eletroanalítica de íons $\mathrm{Na}^{+}$foi feita por voltametria de onda quadrada (VOQ), após otimização dos parâmetros que influenciam a resposta eletroquímica nesta técnica (frequência de aplicação dos pulsos - $f$, amplitude de potencial $-a$, e incremento de varredura $\left.\Delta E_{s}\right)$. As curvas analíticas foram construídas a partir da relação entre a corrente e a concentração do analito, com base no decaimento do pico de oxidação característico do filme de óxido de níquel após sucessivas adições da solução-padrão do analito. Utilizando a equação de regressão linear obtida, foi possível averiguar a precisão e exatidão do método pela determinação da concentração de $\mathrm{Na}^{+}$em soluções padrão deste cátion.

\section{Resultados e Discussão}

\subsection{Modificação do eletrodo}

Conforme descrito anteriormente, o primeiro passo para a construção do EQM de trabalho foi a eletrodeposição do filme de hexacianoferrato de níquel sobre a superfície do eletrodo de pasta de carbono, empregando voltametria cíclica no modo galvanostático. Esta primeira modificação foi feita por varreduras na faixa de 0,0 a $1,0 \mathrm{~V}$ (vs. $\mathrm{Ag} / \mathrm{AgCl} / \mathrm{C}_{\text {saturado }}^{-}$), obtidas a $50 \mathrm{mV} \mathrm{s}^{-1}$, usando uma solução mista, formada por $\mathrm{K}_{3} \mathrm{Fe}(\mathrm{CN})_{6}$ a $1,0 \times 10^{-3} \mathrm{~mol} \mathrm{~L}^{-1}, \mathrm{NiCl}_{2}$ a $1,0 \times 10^{-3} \mathrm{~mol} \mathrm{~L}^{-1}$ e $\mathrm{KCl}$ a $0,2 \mathrm{~mol} \mathrm{~L}^{-1}$, como eletrólito. A modificação do eletrodo é caracterizada pelo surgimento de dois pares redox reversíveis e bem definidos, conforme mostrado na Figura 1. Os dois picos 
de oxidação (picos 1 e 2) e de redução (picos 3 e 4) estão relacionados com as alterações no estado de oxidação dos átomos de ferro presentes no complexo metálico, cujas reações encontram-se representadas nas seguintes equações:

$\left.\mathrm{K}_{2} \mathrm{Ni}^{\mathrm{II}}\left[\mathrm{Fe}^{\mathrm{II}} \mathrm{CN}\right)_{6}\right] \leftrightarrow \mathrm{KNi}^{\mathrm{II}}\left[\mathrm{Fe}^{\mathrm{III}}(\mathrm{CN})_{6}\right]+\mathrm{e}^{-}+\mathrm{K}^{+}$

$\mathrm{KNi}^{\mathrm{II}}{ }_{1.5}\left[\mathrm{Fe}^{\mathrm{II}}(\mathrm{CN})_{6}\right] \leftrightarrow \mathrm{Ni}^{\mathrm{II}}{ }_{1.5}\left[\mathrm{Fe}^{\mathrm{III}}(\mathrm{CN})_{6}\right]+\mathrm{e}^{-}+\mathrm{K}^{+}$

Durante a oxidação, ocorre a oxidação de $\mathrm{Fe}^{2+}$ a $\mathrm{Fe}^{3+}$ e liberação de $\mathrm{K}^{+}$para o seio da solução, enquanto que na redução, o $\mathrm{Fe}^{3+}$ é novamente convertido em $\mathrm{Fe}^{2+}$, juntamente com a absorção de $\mathrm{K}^{+}$a partir da solução (KARNJANAKOM et al., 2014).

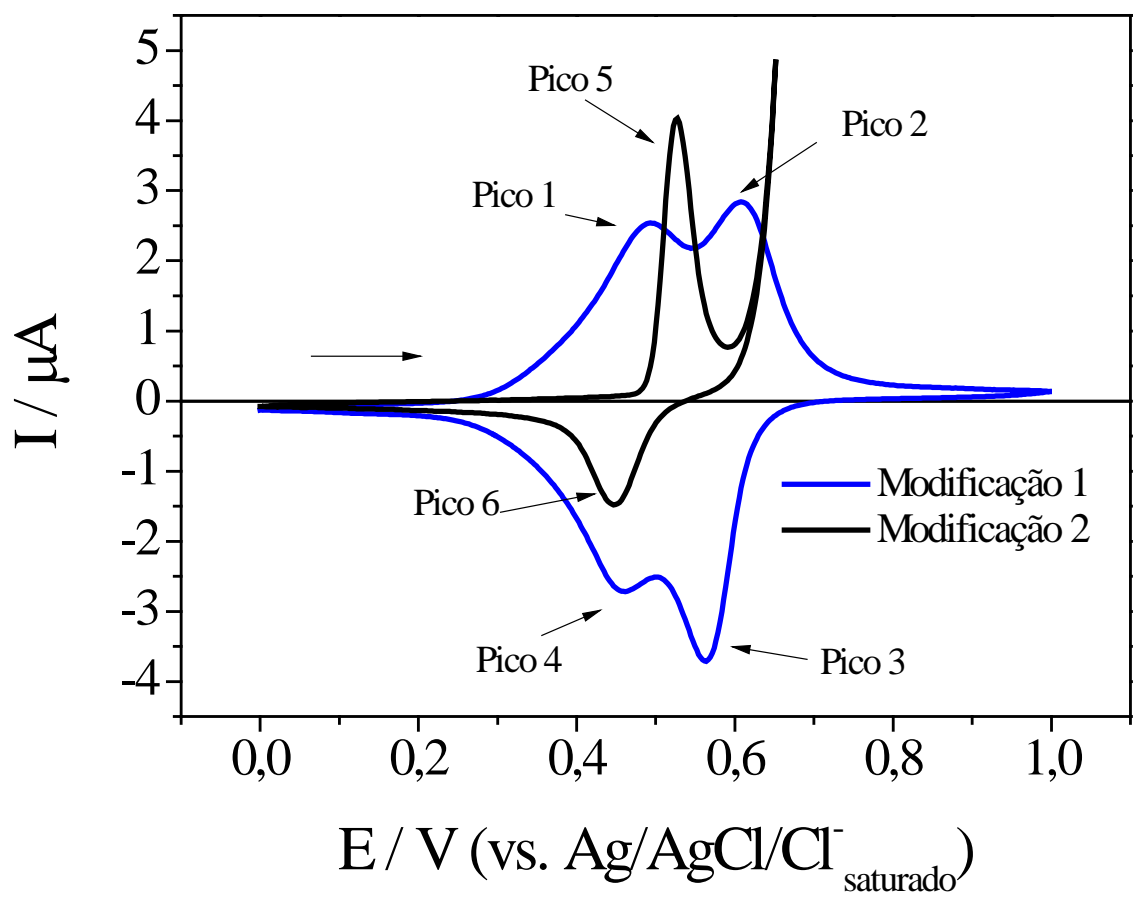

Figura 1- Voltamogramas cíclicos registrados sobre eletrodo de pasta de carbono: (1) modificado com hexacianoferrato de níquel e (2) convertido a óxido de níquel, conforme descrito no item 2.3.

Com o aumento do número de ciclos, há um aumento nas correntes dos picos redox, em virtude do aumento na espessura do filme e da cinética de transferência eletrônica. Entretanto, após 30 ciclos, os valores de corrente aumentaram de forma não-linear, indicando aumento na resistência de transferência eletrônica. Sendo assim, para a eletrossíntese do óxido de níquel, utilizou-se um dispositivo modificado com hexacianoferrato de níquel, obtido após 30 varreduras consecutivas de potencial.

Em meio fortemente alcalino, o filme de óxido de níquel pode ser eletrossintetizado in situ, utilizando o complexo de hexacianoferrato de níquel como precursor. O êxito na obtenção deste novo material na superfície do eletrodo é caracterizado pelo surgimento de um novo processo redox (Modificação 2), cujos picos de oxidação e de redução, representados na Figura 1 como picos 5 e 6, podem ser representados pela seguinte reação:

$\mathrm{Ni}(\mathrm{OH})_{2}+\mathrm{OH}^{-} \leftrightarrow \mathrm{NiOOH}+\mathrm{H}_{2} \mathrm{O}+\mathrm{e}^{-}$

Com o aumento do número de ciclos, este perfil se torna ainda mais intenso, estabilizando-se após 20 ciclagens de potencial consecutivas $(0,0$ a 0,7 V). 


\subsection{Efeito da velocidade de varredura}

Avaliando o efeito da velocidade de varredura (10 e $\left.500 \mathrm{mV} \mathrm{s}^{-1}\right)$ em relação ao perfil voltamétrico do sensor, para uma faixa de potencial entre 0,0 e 0,7 V, também ficou constatado um aumento linear nos valores das correntes de pico $\left(\mathrm{I}_{\mathrm{p}}\right)$ ambos os processos, além do deslocamento dos potenciais de pico $\left(\mathrm{E}_{\mathrm{p}}\right)$ de oxidação para valores mais positivos e no sentido oposto para os picos de redução; uma característica de sistemas difusionais. Após 200 varreduras consecutivas de potencial a $50 \mathrm{mV} \mathrm{s}^{-1}$, em meio de $\mathrm{NaOH} 0,1 \mathrm{~mol} \mathrm{~L}^{-1}$, observou-se um decaimento de apenas $2,7 \%$ do sinal inicial, indicando a alta estabilidade do filme eletrodepositado sobre o eletrodo.

\subsection{Otimização dos parâmetros analíticos}

Em função do modo de amostragem da corrente, as técnicas de pulso como a VOQ possuem menor contribuição de correntes capacitivas e maior sensibilidade para a quantificação dos analitos. Sendo assim, antes da determinação eletroanalítica de íons $\mathrm{Na}^{+}$, os parâmetros da VOQ ( $f, a$ e $\Delta E_{s}$ ) que influenciam a resposta eletroquímica foram otimizados, utilizando o processo anódico do óxido de níquel como sinal analítico, em virtude de sua maior intensidade comparada ao pico de redução. Variando $f$ de 10 a $500 \mathrm{~Hz}$, observou-se deslocamento de $\mathrm{E}_{\mathrm{p}}$ para valores mais positivos, paralelo ao aumento linear de $\mathrm{I}_{\mathrm{p}}$ até $100 \mathrm{~s}^{-1}$. No caso de $a$, avaliada entre 5 e $50 \mathrm{mV}$, os valores de $E_{p}$ para todos os compostos foram deslocados para valores mais positivos com o aumento desta variável, seguido do aumento linear de $\mathrm{I}_{\mathrm{p}}$ até $30 \mathrm{mV}$. Para $\Delta E_{s}$, avaliado entre 1 e $7 \mathrm{mV}$, houve aumento linear de $\mathrm{I}_{\mathrm{p}}$, mas acima de $3 \mathrm{mV}$ ocorreu aumento no ruído do sinal e na largura do processo redox. Com base nos dados obtidos, utilizou-se $f=100 \mathrm{~Hz}, a=30 \mathrm{mV}$ e $\Delta E_{s}=3 \mathrm{mV}$ como condição otimizada.

\subsection{Determinação eletroanalítica}

Utilizando NiOx/EPC e VOQ sob condições otimizadas, observou-se que o aumento da concentração de íons $\mathrm{Na}^{+}$no meio promove a intrusão deste cátion no retículo do filme, causando uma diminuição não-linear nos valores de $\mathrm{I}_{\mathrm{p}}$, conforme apresentado na Figura 2A.

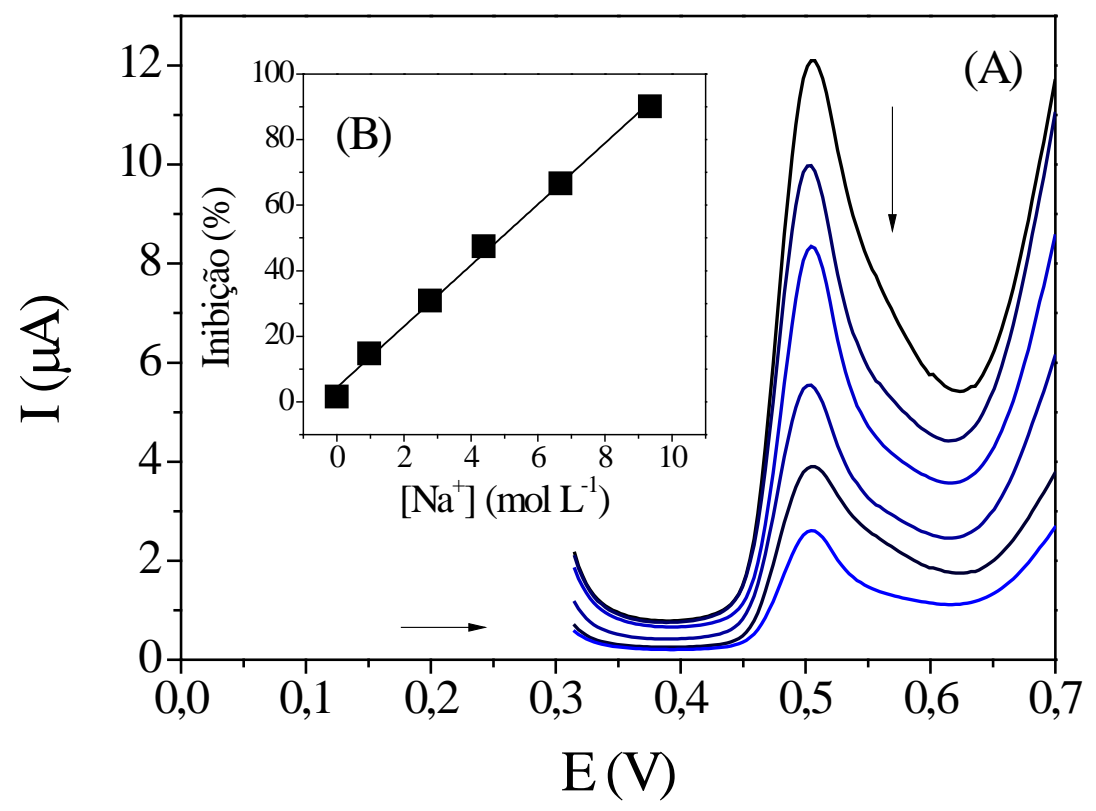

Figura 2- (A) Voltamogramas de onda quadrada registrados sobre eletrodo de pasta de carbono modificado com óxido de níquel, após adições crescentes de íons sódio $\left(9,72 \times 10^{-7}\right.$ a 9,34×10-6 $\left.\mathrm{mol} \mathrm{L}^{-1}\right)$, 
empregando $f=100 \mathrm{~Hz}, a=30 \mathrm{mV}$ e $\Delta E_{s}=3 \mathrm{mV}$, em meio de $\mathrm{NaOH} 0,1 \mathrm{~mol} \mathrm{~L}^{-1}$. (B) Curva analítica referente às adições do analito.

Foi observada uma relação linear entre a porcentagem de inibição do sinal em função da concentração do cátion, para uma faixa de $9,72 \times 10^{-7}$ a $9,34 \times 10^{-6} \mathrm{~mol} \mathrm{~L}^{-1}$, com limite de detecção de $9,50 \times 10^{-7} \mathrm{~mol} \mathrm{~L}^{-1}$ e um coeficiente de correlação equivalente á 0,9962, o que sugere uma boa correlação entre os dados. A equação de regressão linear, obtida para a curva analítica apresentada na Figura 2B, pode de descrita como:

$$
\text { Inibição }(\%)=4,37+9,32 \times 10^{6} \times\left[\mathrm{Na}^{+}\right]\left(\mathrm{mol} \mathrm{L}^{-1}\right)
$$

A precisão das medidas foi constatada por testes de repetibilidade, para uma concentração de $5,00 \times 10^{-6} \mathrm{~mol} \mathrm{~L}^{-1}$ do analito, resultando em um desvio padrão relativo de $5,50 \%$. Em relação à reprodutibilidade, analisada pelas medidas de três diferentes dispositivos construídos sob as mesmas condições otimizadas, o desvio padrão relativo foi equivalente a $6,71 \%$. De um modo geral, o conjunto de dados analíticos obtidos mostram que NiOx/EPC pode ser uma ferramenta promissora para a análise de $\mathrm{Na}^{+}$em diferentes matrizes ambientais.

\title{
4. Conclusões
}

O método eletroanalítico proposto, empregando NiOx/EPC como sensor de trabalho associado à VOQ, mostrou-se eficiente para a detecção e quantificação de íons $\mathrm{Na}^{+}$. Entre as principais vantagens do sensor, destacam-se sua alta estabilidade e sensibilidade para fins analíticos. Os baixos valores de desvio padrão relativo obtidos nos testes de precisão e exatidão das medidas reforçam que NiOx/EPC pode ser uma ferramenta analítica promissora para a análise de íons $\mathrm{Na}^{+}$em amostras complexas.

\section{Electroanalytical determination of sodium ions using a nickel oxide modified carbon paste electrode}

\begin{abstract}
A supplemented diet with $\mathrm{Na}^{+}$ions has been linked to heart disease, hormone disorders, allergies, and other pathologies. Conventionally, the monitoring of this cation in different matrices has been done by chromatographic and spectroscopic methods, although they present limitations regarding the cost, interference and analysis time. This work aims to develop an alternative electroanalytical method using a nickel oxide chemically modified carbon paste electrode (NiOx/EPC), which allows the detection of this cation in different matrices and presents a better cost-benefit ratio if compared to the conventional methods. Square-wave voltammetry (SWV) was used for the quantification step, after optimization of the parameters that control the electrochemical response in this technique (frequency of $100 \mathrm{~Hz}$, amplitude of

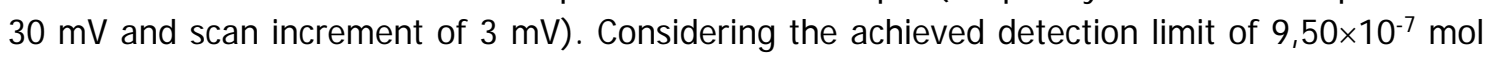
$\mathrm{L}^{-1}$ and low relative standard deviation for measurements precision tests $(<7.0 \%)$, the association between NiOx/EPC e VOQ can provide promising results on electroanalysis and quality control of supposed contaminated samples by this cation.
\end{abstract}

Keywords: Sodium ions; Chemically modified electrodes; Nickel oxide; Electroanalytical determination.

\section{Referências Bibliográficas}

ABBASPOUR, A.; KHAJEHZADEH, A.; GHAFFARINEJAD, A. Electrocatalytic oxidation and determination of hydrazine on nickel hexacyanoferrate nanoparticles-modified carbon ceramic electrode. Journal of Electroanalytical Chemistry, v. 631, p.52-57, 2009. 
APHA. Standard Methods for the Examination of the Water and Wastewater. 19. ed. New York, USA: American Public Health Association, 2005.

BUFFON, E.; VASCONCELLOS, M. L. S.; PROFETI, D.; PROFETI, L. P. R. Influência da composição de filmes de hexacianoferrato de metais na eletro-oxidação de ácido ascórbico. In: ENCONTRO CIENTÍfICO DE FÍSICA APLICADA, 6., 2015, São Paulo. Anais... São Paulo: Blucher Proceedings, 2015.

CARMO, D. R.; SILVA, R. M.; STRADIOTTO, N. R. Electrocatalysis and Determination of Ascorbic Acid Through Graphite Paste Electrode Modified With Iron Nitroprusside.

Portugaliae Electrochimica Acta, Araraquara, SP, v.22, n. 2, p.71-79, 2004.

CASTILHO, M. S.; STRADIOTTO, N. R. Determination of potassium ions in biodiesel using a nickel (II) hexacyanoferrate-modified electrode. Talanta, v. 74, n. 5, p. 1630-1634, 2008.

CHAUDHURI, S.; ALE, S. Long term (1960-2010) trends in groundwater contamination and salinization in the Ogallala aquifer in Texas. Journal of Hydrology, Vernon, TX , v. 513 p. 376-390, 2014.

CHEN, W.; TANG, J.; CHENG, H.-J.; XIA, X.-H. A simple method for fabrication of sole composition nickel hexacyanoferrate modified electrode and its application. Talanta, v. 80, p. 539-543, 2009.

FIRMINO, M. C.; FARIAS, M. S. S. de; MEDEIROS, S. S. de; CARVALLO, H. O. G.; GUIMARÃES, J. P. Características químicas do solo influenciadas pela adição de água residuárias tratada sob cultivo do pinhão manso. Agropecuária Científica no Semiárido, Patos, PB, v. 11, n. 2, p. 32-37, 2015.

HIN, Y. B. F.; LOWE, C.R. Catalytic oxidation of reduced nicotinamide adenine dinucleotide at hexacyanoferrate-modified nickel electrodes. Analytical Chemistry, United Kingdom, v. 59, p. 2111, 1987.

KARNJANAKOM, S.; MA, Y.; GUAN, G.; PHANTHONG, P.; HAO, X.; DU, X.; SAMART, C.; ABUDULA, A. Fabrication of nickel hexacyanoferrate film on carbon fibers by unipolar pulse electrodeposition method for electrochemically switched ion exchange application.

Electrochimica Acta, v. 139, p. 36-41, 2014.

KELLY, W.R.; PANNO, S.V.; HACKLEY K.C. Impacts of road salt runoff of the Chicago, Illinois, region. Environmental and Engineering Geoscience, Champaign, IL, v. 18 p. 65-81, 2012.

MACHIWAL, D.; JHA, M.K. Identifying sources of groundwater in a hard-rock aquifer systemusing multivariate statistical analyses and GIS-based geostatistical modeling techniques. Journal of Hydrology: Regional Studies, 2015, DOI:

http://dx.doi.org/10.1016/j.ejrh.2014.11.005

MAFTALE, T.J.; NYOKONG, T. Electrocatalytic Oxidation of Cysteine by molybdenum (V) phthalocyanine complexes. Journal of Electroanalytical Chemistry, v. 408, n. 1, p. 213-218, 1996.

MOSES, P.R.; WIER, L.; MURRAY, R.W. Chemically Modified Tin Oxide Electrode. Analytical Chemistry, Chapel Hill, NC, v. 47, n.12, p. 1882-1886, 1975. 
OLIVEIRA, T.M.B.F. Biossensores enzimáticos para deteç̧ão e quantificação de carbonato em amostras de alimentos. 2013. 131f. Tese (Doutorado em Química- Química Analítica) Programa de Pós-Graduação em Química, Universidade Federal de Ceará, Fortaleza-CE, 2013.

PEREIRA, A. C., SANTOS, A. S., KUBOTA, L. T. Tendências em modificação de eletrodos amperométrico para aplicações eletroanalíticas. Química Nova, Campinas, SP, v.25, n.6, p.1012-1021, 2002.

QUINTINO, M. S. M. Desenvolvimento de sensores eletroquímicos associados a batch injection analysis (bia) para aplicações analíticas. 2003. 112f. Tese (Doutorado em Ciências Química Analítica) - Instituto de Química, Universidade de São Paulo, 2003.

SCHNEEMEYER, L. F.; SPENGLER, S. E.; MURPHY, D. W. Ion selectivity in nickel hexacyanoferrate films on electrode surfaces. Inorganic Chemistry, New Jersey, v.24, p. 30443046, 1985.

SHEN, G.; YANG, M.; YANG, Y.; QU, F.; LU, Y.; YU, R. Attachment of nickel hexacyanoferrates nanoparticles on carbon nanotubes: Preparation, characterization and bioapplication. Analytical Chemistry Acta, v. 571, p. 211-217, 2006.

SOUZA, M.F.B. Eletrodos quimicamente modificados aplicados à eletroanálise: uma breve abordagem. Química Nova, São Paulo, v. 20, p. 191-195, 1997.

VINCENTINI, F.C. Avaliação da potencialidade de eletrodos compósitos a base de grafite/poliuretana modificados com hexacianoferrato de Cu (II), Co (II) e Fe (III) para fins analíticos. 2009. 101f. Dissertação (Mestrado em Química)- Instituto de Química de São Carlos, Universidade de São Paulo, São Carlos, 2009. 